\title{
Del lenguaje a lalangue: cruces entre el psicoanálisis y la lingüística
}

\section{From Language}

to Lalangue:

Crossings between

Psychoanalysis and

Linguistics
Da linguagem

à lalangue:

cruzamentos entre

psicanálise e

linguística
Primer semestre de 2021 - pp. 45-56

Segunda época

N.

Savio, K. (2021). Del lenguaje a lalangue: cruces entre el psicoanálisis y la lingüística. Folios, 53. https:/doi.org/10.17227/folios.53-10927

Artículo recibido $13 \cdot 12 \cdot 2019$

Artículo aprobado $08 \cdot 06 \cdot 2020$

Doctora en letras (área linguística). CONICET-uBA-UNAJ.

Correo electrónico: karinasavio@fibertel.com.ar 


\section{Resumen}

Este artículo de reflexión indaga las nociones de lengua, lenguaje y lalangue en las enseñanzas de Lacan. En primer lugar, interroga el término lenguaje en la definición de inconsciente. Por ello, problematiza la distinción entre lenguay lenguaje en los desarrollos teóricos de Saussure (2007), Benveniste (2004) y Jakobson (1985). En segundo lugar, estudia el neologismo lalangue en este discurso para pensar qué matices agrega este término. Como resultado de este trabajo, se advierte que la noción de lenguaje es más adecuada que la de lengua para aludir al inconsciente. En efecto, el lenguaje es considerado un fenómeno complejo, que no resulta aprehensible con facilidad. Sobre la lalangue, se evidencian cinco ejes con los que se articula: los equívocos, el inconsciente, la interpretación, el laleo y el síntoma.

\section{Palabras clave}

lenguaje; lengua; inconsciente; discurso

\section{Abstract}

This reflective article inquires the notions of language, speech and lalangue in Lacan's teachings. First, it interrogates the term language in the definition of the unconscious. Therefore, it problematizes the distinction between language and speech in the theoretical developments of Saussure (2007), Benveniste (2004) and Jakobson (1985). Second, it studies the lalangue neologism in this discourse to think about the nuances this term adds. As a result of this work, it is noted that the notion of language is more appropriate than speech to refer to the unconscious. Indeed, language is considered a complex phenomenon, which is not easily apprehended. Referring to the lalangue, five axes with which it is articulated are evident: the misunderstandings, the unconscious, the interpretation, the lalation and the symptom.

\section{Keywords}

language; speech; unconscious; discourse

\section{Resumo}

Este artigo de reflexão investiga as noções de língua, linguagem e lalangue nos ensinamentos de Lacan. Primeiro, ele interroga o termo linguagem na definição do inconsciente. Portanto, problematiza a distinção entre língua e linguagem nos desenvolvimentos teóricos de Saussure (2007), Benveniste (2004) e Jakobson (1985). Segundo, estuda o neologismo lalangue nesse discurso para pensar sobre quais nuances esse termo adiciona. Como resultado deste trabalho, nota-se que a noção de linguagem é mais adequada do que a noção de língua para se referir ao inconsciente. De fato, a linguagem é considerada um fenômeno complexo, que não é facilmente apreendido. Na lalangue, são evidentes cinco eixos com os quais se articula: 0 ambíguo, o inconsciente, a interpretação, o laleo e o sintoma.

\section{Palavras chave}

linguagem; língua; inconsciente; discurso 


\section{Introducción}

Lengua, lenguaje, discurso son tres términos que, entre otros, a mediados de los cincuenta, y a lo largo de los años sesenta, comenzarán a forjar nuevos sentidos que renovaron los estudios del lenguaje y que, en este mismo movimiento, revolucionaron sus bases teóricas. Estos nuevos alcances no son ajenos a Lacan quien, como es conocido, acatando a la letra freudiana, tejió un psicoanálisis a partir de saberes provenientes de diferentes campos de estudio, entre ellos, los elaborados por la lingüística. El texto "Función y campo de la palabra y del lenguaje en psicoanálisis" (Lacan, 2002), escrito tempranamente en 1953, inauguró así un camino que, a pesar de que se resignificó y, por ende, se modificó a lo largo de los años, continuó vigente hasta sus últimos trabajos. La tríada lengua, lenguaje y discurso adoptó, entonces, un valor medular en la trama de las enseñanzas lacanianas, a la que se sumó, a partir de 1971, la noción de lalangue. ${ }^{1}$

Desde sus inicios, la clínica psicoanalítica se concibió como una cura por medio de la palabra -talking cure-, cuyo correlato teórico encontró su sustento en dos aforismos de Lacan: el inconsciente es el discurso del Otro y el inconsciente está estructurado como un lenguaje. El estatuto del inconsciente quedó, desde allí, asociado a la materialidad lingüística, lo que contribuyó al acercamiento entre la teorización psicoanalítica y los diversos saberes acerca del lenguaje. En estas páginas nos proponemos trabajar esta articulación, en particular, el par lengua-lenguaje y su relación con lalangue. Nos interesa, por un lado, revisar qué se entiende por lengua y por lenguaje en esos años, a partir de aquellos enfoques lingüísticos a los que Lacan recurre para sus formalizaciones teóricas y, por el otro, reflexionar qué aporta el novedoso término lalangue a los desarrollos teóricos psicoanalíticos y a aquellos relativos al campo del lenguaje.

1 Para este trabajo mantenemos el uso del neologismo en francés. Como veremos más adelante, esta noción nace de un equívoco y establece lazos homofónicos con una serie de términos próximos. Por ello, hemos decidido utilizar el original y no incluir su traducción al español (lalengua).
Cuando comenzamos este recorrido -la búsqueda de lengua, lenguaje y lalangue en los textos lacanianos-, se nos impuso una pregunta cuyo eco encontramos también en un artículo de Arrivé (2000). El psicoanalista francés, ya lo hemos recordado, afirma que el inconsciente está estructurado como un lenguaje, premisa que nace conceptualmente a partir del Curso de lingüística general de Saussure (2007). No obstante, para ser rigurosos, este lingüista plantea -o así nos lo han hecho llegar sus discípulos- que es la lengua aquella que conforma sistema y no el vasto fenómeno del lenguaje. ¿Por qué, entonces, Lacan opta por emplear el término langage (lenguaje) y no langue (lengua) para remitirse al inconsciente? Arrivé (2000) responde que el psicoanalista utiliza la noción de lenguaje con el sentido de lengua, como su sinónimo, y que, por esa razón, emplea el artículo "un". En este trabajo, sin embargo, nos guiamos a partir de otra hipótesis, por lo que buscamos precisar y completar esta respuesta a partir de estudiar qué entienden por lengua y lenguaje Saussure, Benveniste y Jakobson, ${ }^{2}$ interlocutores de Lacan, para pensar esta relación. Luego, pretendemos reflexionar y analizar qué es lo que la noción de lalangue añade.

Antes de comenzar este trayecto, nos interesa marcar que, a pesar de los estrechos vínculos entre la lingüística y el psicoanálisis, no han sido muchos los lingüistas que han ahondado en las enseñanzas lacanianas para revisarlas en relación con el campo de los estudios del lenguaje. En efecto, la pregunta respecto a qué es lo que el psicoanálisis lacaniano le aporta a esta área del saber no ha sido aún abordada en profundidad. ${ }^{3}$ Entre las elaboraciones que buscan iluminar esta zona en común no podemos dejar de mencionar las de Milner (1980, 2003) y Arrivé (2001). Sin embargo, en Argentina esta línea de investigación es aún incipiente y se

2 No incluimos en esta lista a Noam Chomsky (1997), ya que en el momento en el que Lacan articula inconsciente y lenguaje, a partir del enfoque estructuralista de la lengua, este lingüista no es un interlocutor directo de sus enseñanzas.

3 No nos referimos aquí a la línea del análisis del discurso que se inaugura con Pechêux y que ancla sus bases, entre otros campos del saber, en el psicoanálisis lacaniano. Aludimos aquí a la lectura detenida de los textos lacanianos desde una mirada lingüística. 
encuentra en formación. ${ }^{4} \mathrm{~A}$ los fines de problematizar términos que muchas veces aparecen cristalizados en estudios lingüísticos, en presentaciones anteriores nos hemos dedicado a reflexionar sobre las nociones de discurso (Savio, 2015), sujeto de la enunciación (Savio, 2017) y escritura (Savio, 2019) a la luz de los escritos, de las conferencias y de los seminarios de Lacan.

Este artículo está organizado en dos partes. En la primera de ellas revisamos las definiciones de lengua y lenguaje desde las perspectivas de Saussure, Benveniste y Jakobson con el objetivo de interrogar y, en esta misma operación, precisar la proposición lacaniana que afirma que el inconsciente está estructurado como un lenguaje; buscamos identificar las razones por las que el término lenguaje le resulta a Lacan más cómodo y adecuado para remitir al inconsciente en lugar de lengua. En la segunda, perseguimos la aparición de la noción de lalangue en las enseñanzas lacanianas; intentamos en esta segunda instancia pensar qué matices agrega este término y cuáles podrían ser sus implicancias en los estudios lingüísticos.

\section{El par lengua/lenguaje en Saussure, Benveniste y Jakobson}

Es claro y conocido el interés de Saussure por definir la lengua (langue) y así delimitar el objeto de estudio de la lingüística. Para ello, la distingue del lenguaje (langage) y del habla (parole). En términos generales, en el Curso de lingüística general ${ }^{5}$ (Saussure, 2007) se señala que el lenguaje tiene un lado individual y uno social y que la lengua es solo una de las manifestaciones del lenguaje. Es, en efecto, su parte social. En tanto existe en la masa y es exterior al individuo, este no puede ni crearla ni cambiarla. De esta manera, es un "producto" (Saussure, 2007) que se registra pasivamente. La lengua es aquí entendida como sistema de valores puros, en donde todos los

4 Como excepción, podemos mencionar el caso brasilero, en el que este terreno ha comenzado a florecer en los últimos años (Flores, 1999).

5 En estas páginas abreviaremos el nombre de este texto y lo denominaremos el Curso. términos son solidarios y donde el valor de cada uno resulta de la presencia simultánea de los otros. Es, por ello, el domino de las articulaciones.

A diferencia de la lengua, el habla es pensada y definida como un acto individual de voluntad e inteligencia. Sus manifestaciones son, en este sentido, accidentales y momentáneas. Por tal razón, no constituye, según Saussure, un objeto que pueda estudiarse separadamente. En otras palabras, el habla no puede conformarse como el objeto de la lingüística.

A pesar de que esta diferenciación ha sido retomada y desarrollada en un sinfín de trabajos. En este artículo quisiéramos puntualizar dos aspectos de esta caracterización que nos permitirán, con posterioridad, ubicar la pregunta por el inconsciente: el carácter heteróclito del lenguaje, por un lado, y la unidad de la lengua, por el otro.

En primer lugar, en el Curso el lenguaje es entendido como multiforme y heteróclito:

a caballo en diferentes dominios, a la vez físico, fisiológico y psíquico, pertenece además al dominio individual y al dominio social; no se deja clasificar en ninguna de las categorías de los hechos humanos ${ }^{6}$, porque no sabe cómo desembrollar su unidad. (Saussure, 2007, T.I, p. 57)

En este fragmento nos encontramos con que el lenguaje aparece asociado a lo inclasificable; es decir, es un fenómeno que escapa a toda tipificación. No puede ser aprehendido por una única categoría porque no pertenece a ninguna clase: es individual y es social; es, a su vez, físico, fisiológico y psíquico. Por ende, su "unidad" no puede desenredarse.

Este aspecto también diferencia el lenguaje de su concepción de lengua. En efecto, en estas páginas se destaca que, mientras que la lengua en tanto sistema de signos presenta una naturaleza homogénea, el lenguaje es, por el contrario, heterogéneo (Saussure, 2007). Es en este sentido que la lengua se constituye como un objeto pasible de ser delimitado:

6 A menos que se señale las cursivas son siempre nuestras. 
es un objeto bien definido en el conjunto heteróclito de los hechos de lenguaje. (Saussure, 2007, T.I, p. 64)

En esta cita se advierte el contraste cómo la lengua y el lenguaje son mencionados: la lengua es entendida en tanto objeto, en tanto entidad; el lenguaje, por su parte, en tanto conjunto de hechos.

En segundo lugar, a la lengua se la caracteriza como un sistema completo y localizable:

La lengua, por el contrario, es una totalidad en sí y un principio de clasificación. (Saussure, 2007, T. I, p. 58)

es la lengua la que hace la unidad del lenguaje. (Saussure, 2007, T. I, p. 60)

se la puede localizar en la porción determinada del circuito donde una imagen acústica viene a asociarse con un concepto. (Saussure, 2007, T. I, p. 64)

De esta forma, la lengua se constituye como una totalidad y puede ser clasificada y categorizada. Es, por esta razón, aquella que otorga unidad dentro de la heterogeneidad del lenguaje. Incluso puede ser localizada y puede ubicarse en un lugar del circuito de la palabra, en el punto en el que la imagen acústica se asocia con un concepto.

Mientras que la teoría saussureana distingue, como hemos recordado, lengua, lenguaje y habla, podría pensarse que Benveniste reformula esta tríada y, en su lugar, ubica la lengua, el lenguaje y el discurso. En esta serie la enunciación quedará situada en línea con el discurso.

En "Ojeada al desenvolvimiento de la lingüística" de 1963 Benveniste (2004b) afirma que lingüística presenta un doble objeto: es ciencia del lenguaje y ciencia de las lenguas. El lenguaje se define, para él, como una facultad humana, una característica universal e inmutable y las lenguas, por su parte, son individuales y variables, son aquellas que lo realizan. Estudiar las lenguas y analizar lo que tienen en común implica, entonces, dar cuenta del lenguaje. De esta forma, es necesario alcanzar cierto grado de generalidad en el estudio de las lenguas para poder caracterizar el lenguaje en tanto tal. Si bien en varios artículos de Benveniste esta diferenciación no siempre se sostiene de manera taxativa, ya que en distintos trabajos utiliza la noción de lenguaje como sinónimo de lengua, se evidencia que en la mayoría de los casos la lengua queda definida en términos estructurales y que el lenguaje, en cambio, queda asociado a sus efectos.

A través de la mediación del lenguaje la realidad es (re)producida de nuevo, aunque sometiéndola a una nueva organización. Es, en este sentido, que Benveniste (2004a) hace referencia a su "poder fundador". Esta concepción se aleja de la perspectiva instrumental de la palabra: el lenguaje no es una herramienta; sus propiedades, su naturaleza inmaterial y articulada, su funcionamiento simbólico, lo alejan de la simplificación que conlleva esta visión. Pero, además, el lenguaje no solamente produce el acontecimiento - y lo reproduce-, sino que también es aquello que constituye al hombre como sujeto. El lenguaje es, pues, "la posibilidad de la subjetividad" (Benveniste, 2004a, T.I, p. 184), funda en su realidad el concepto "ego".

Es interesante señalar que en una entrevista para Le Nouvel Observateur Benveniste describe el carácter efímero del lenguaje:

La lingüística es el intento de apresar ese objeto evanescente que es el lenguaje. (2004a, T.II, p. 32)

Como vemos, a diferencia del Curso, el lenguaje es pensado aquí como un objeto. No obstante, es un objeto que se esfuma, que se escapa, que no puede ser aprehendido en su totalidad.

La lengua, por otra parte, es aquella que forma sistema a partir de la relación y oposición entre los signos lingüísticos: es así una estructura lingüística “definida y particular" (Benveniste, 2004a, T.r, p. 31). Se compone, por ello, de elementos formales y discretos, articulados en diversas combinaciones variables, según ciertos principios de estructura.

En 1967, en "La forma y el sentido en el lenguaje", Benveniste (2004c) diferenció dos modalidades de la lengua, una semiótica y una semántica, que se corresponden con dos funciones lingüísticas: la de significar y la de comunicar. La primera de ellas está relacionada con el signo lingüístico y con la propiedad de la lengua entendida como sistema. Por el contrario, lo semántico se articula con el dominio 
de la lengua en uso y en acción, es el resultado de la actividad del locutor que pone "en acción la lengua”. Esta segunda vertiente es aquella que está vinculada con la noción de discurso.

Finalmente, para el lingüista, el discurso es la lengua en tanto que asumida por el hombre que habla y es la enunciación la que permite esta conversión. Antes de la enunciación, la lengua "no es más que la posibilidad de la lengua" (Benveniste, 2004a, T.II, p. 84). Después de la enunciación, la lengua se efectúa en una instancia de discurso, que nace de un locutor y que causa una enunciación a cambio. De esta manera, la enunciación es definida como aquello que pone a funcionar la lengua por un acto individual de utilización. Esta apropiación a través del discurso permite, según Benveniste, la emergencia de la subjetividad.

En cuanto a Roman Jakobson, nos interesa detenernos brevemente en uno de sus textos clásicos, dado que es citado por Lacan en reiteradas oportunidades. Nos referimos al artículo "Lingüística y poética" de 1960 que nace de una ponencia enunciada unos años previos en una universidad norteamericana. Antes de desarrollar este punto es necesario recordar que el original de este trabajo está escrito en inglés, por lo que la diferencia entre lengua y lenguaje queda neutralizada bajo el término language. ${ }^{7}$

En “Lingüística y poética”, Jakobson (1985) refiere a las distintas funciones que convergen en el lenguaje a partir de los seis factores básicos que lo componen: destinador, destinatario, contexto, mensaje, contacto y código. En efecto, para este lingüista, en un acto de comunicación verbal el destinador manda un mensaje al destinatario y para que este sea operante requiere de un contexto de referencia que el destinatario pueda captar. Asimismo, afirma que es necesario un código común entre ambos participantes del hecho discursivo y un contacto, es decir, un canal físico y una conexión psicológica entre el destinador y el destinatario que les permita sostener la comunicación.

Según Jakobson, cada uno de estos factores determina una función diferente del lenguaje. Por

7 En las traducciones al inglés del Curso se suelen usar los términos language, speech, speaking para referirse a la tríada saussureana. esta razón, distingue seis funciones que aparecen entrecruzadas y que se vinculan con cada factor: una función referencial, en la que predomina la orientación al contexto; una función emotiva o "expresiva", centrada en el destinador; una función conativa, orientada hacia el destinatario; una función fática, orientada hacia el contacto; una función metalingüística, centrada en el mismo código y una función poética, orientada hacia el mensaje.

Ahora bien, de este esquema podemos destacar dos aspectos que están relacionados con el tema que nos convoca. Por un lado, se observa que el lenguaje no puede reducirse a un único elemento, a una única variable. En efecto, en la teoría jakobsiana el lenguaje está asociado a seis factores diferentes que tienen una naturaleza compleja y diversa. Tampoco presenta una única función. Por el otro, se advierte que la lengua entendida en tanto sistema puede alojarse en el lugar del código, aquello que el destinador y el destinatario tienen en común. La lengua es, entonces, solo uno de los factores que intervienen en la comunicación, pero no es el único.

Luego de este recorrido por aquellos enfoques con los que Lacan dialoga en sus enseñanzas, volvamos a la definición del inconsciente y su articulación con el lenguaje. Al comienzo de nuestro artículo habíamos señalado que para el psicoanalista francés el inconsciente está estructurado como un lenguaje. Veamos lo que plantea en 1976 en "De James Joyce como síntoma”:

Lo esencial que por otra parte estoy lejos de haber resuelto, al hablar de un lenguaje; yo he dicho un lenguaje porque parece justamente que, en todo lo que existe del orden de la lengua, haya algo común; algo común que es una alta abstracción, que es que cada lengua tiene una sintaxis. ${ }^{8}$ (Lacan, 1976a, pp. 18-19)

En esta cita encontramos que Lacan distingue lengua y lenguaje con un criterio similar al de Benveniste. El lenguaje es pensado en este fragmento como el punto en común entre cada lengua y en ese punto ubica la sintaxis.

8 El destacado es del original. 
Pero, además de lo que el mismo psicoanalista apunta, a partir de lo que expusimos en este apartado en relación con los enfoques lingüísticos que circulan en Francia en esos años, podría plantearse que el término lenguaje es más adecuado y preciso que la noción de lengua para aludir al inconsciente. En efecto, el lenguaje es considerado un fenómeno complejo que no resulta aprehensible con facilidad. No puede ser pensado como una unidad ni como una totalidad cerrada y localizable. Es heterogéneo, multifactorial e inclasificable. Tiene un carácter universal y es inmutable. Es, asimismo, lo que para Benveniste constituye al hombre como sujeto y lo que permite organizar su "realidad". Es interesante advertir que Lacan describe el inconsciente como evanescente, al igual que lo hace Benveniste con respecto al lenguaje:

La aparición evanescente sucede entre los dos puntos, el inicial, el terminal, de este tiempo lógico: entre ese instante de ver donde algo se elide siempre, se pierde incluso, en la intuición misma, $y$ ese momento elusivo en que, precisamente, la aprehensión del inconsciente con concluye, en que se trata siempre de una recuperación engañosa.

Así pues, ónticamente, el inconsciente es lo evasivo. (Lacan, 1987, p. 40)

Por su parte, las definiciones de lengua que hemos introducido aquí se alejan de la concepción de inconsciente en Lacan. La lengua se presenta como un sistema completo, ubicable y homogéneo. Es variable y común a los individuos. Podría pensarse que es lo que permite la comunicación entre ellos. Constituye una unidad - puede ser definida y delimitada - y, por este motivo, puede ser entendida como un objeto de estudio que puede ser estudiado por la ciencia.

En suma, consideramos que la noción de lenguaje es más apropiada que la de lengua, ya que es pasible de ser homologada a la del inconsciente lacaniano. En efecto, mientras que la lengua se constituye como Una, el lenguaje se presenta desde la falta, desde la imposibilidad de conformar una totalidad, una entidad y escapa, por ello, a cualquier intento de clasificación.

\section{Lalangue}

El 4 de noviembre de 1971 es la primera vez que Lacan (2012) hace referencia a lalangue a partir de un tropiezo en su propio discurso. En esta conferencia, en la que se despliega, entre otros temas, la cuestión del saber, el psicoanalista, al recordar su frase "El inconsciente está estructurado como un lenguaje", comete un lapsus relacionado con el Diccionario de Psicoanálisis publicado por Laplanche y Pontalis, ya que en su lugar hace referencia al Vocabulario de filosofía de Lalande. Y, a partir del malentendido de un oyente que repregunta ‘'lalangue?', Lacan se apropia del neologismo y escribe lalangue en una sola palabra. Allí afirma: "No dije que el inconsciente esté estructurado como lalengua, sino como un lenguaje" (Lacan, 2012, p. 23). A partir de esta premisa, diferencia lo que es lalangue de lo que es el diccionario y vincula el inconsciente con la gramática y la repetición.

En esta misma conferencia Lacan establece una definición del lenguaje. En efecto, aquí plantea que el lenguaje trata de rasgos comunes que se encuentran en lalangue: "Aunque esta misma está sujeta a una gran variedad, sin embargo tiene constantes" (Lacan, 2012, p. 30). Por ello, utilizando términos jakobsianos, ubica el lenguaje en el lugar del código.

En la siguiente charla, la del 2 de diciembre de 1971, el psicoanalista retoma la noción de lalangue y la vincula con lo real y con el déficit. En este punto, refiere que es una dimensión de la castración (Lacan, 2012, p. 70), por lo que podría caracterizarse como no-toda. ${ }^{9}$

Numerosos estudios se han publicado desde el campo psicoanalítico con el objetivo de dar cuenta del término lalangue. Entre ellos, podemos mencionar "La teoría de lalengua", conocida exposición de Miller (1987) ante el Congreso de la Escuela Freudiana en $1974 .{ }^{10}$ Por su parte, desde la lingüística también encontramos trabajos que bordean o analizan este neologismo. En este sentido, los

9 En este sentido, podría leerse lo que Lacan plantea el 8 de marzo de 1972 cuando afirma que lalangue es "generosa, pero no tanto" (Lacan, 2016, p. 111).

10 Otros trabajos más recientes son los de Dahan (2011), Godoy (2016), Rouillon (2011). 
artículos de Arrivé (2000), Henry $(2012,2019)$ y Milner (1980) problematizan distintos aspectos del lenguaje y refieren de diferentes maneras a lalangue. Mientras que Arrivé (2001) —quien pone el foco en la gramática - señala brevemente que lalangue remite a la homofonía y al equívoco, Henry (2012, 2019) está interesado en revisar las implicancias del psicoanálisis lacaniano sobre el equívoco para la lingüística y el análisis del discurso. No obstante, es importante destacar que no realiza un recorrido por las enseñanzas de Lacan en las que se emplea esta noción. Milner (1980) es, en cambio, quien se detiene con mayor detalle en lalangue en El amor por la lengua. En este texto define esta noción y la caracteriza de diversas formas: lalangue es, en toda lengua, aquel registro que la consagra al equívoco; es no-toda; es aquello por lo cual un ente puede ser nombrado hablente; es una multiplicidad de arborescencias sobreabundantes, donde el sujeto fija su deseo; es el conjunto virtual de los decires del deseo.

Ahora bien, en este apartado buscamos retornar a la letra lacaniana sobre lalangue, para lo cual hemos realizado una lectura atenta sobre la totalidad de las referencias que el psicoanalista realiza en torno a esta noción. ${ }^{11}$ De este trayecto quisiéramos marcar dos aspectos que consideramos relevantes para reflexionar sobre ella. En primer lugar, advertimos que, a diferencia de lo que ocurre con otros términos lacanianos, no se introducen giros teóricos sustanciales respecto a lo que se entiende por lalangue. En efecto, en el último tramo de las enseñanzas de Lacan se evidencia cierta continuidad en relación con su conceptualización en los seminarios, conferencias y escritos en los que la menciona. Sin embargo, encontramos que los distintos usos del término reenvían a diferentes vertientes de la noción que, como planteamos, no son necesariamente contradictorias. En segundo lugar, es posible ubicar cinco ejes con los que esta noción se articula: los equívocos o tropiezos del lenguaje, el inconsciente, la interpretación, el laleo y el síntoma. Estos ejes no pueden entenderse ni leerse de manera separada,

11 Quisiéramos destacar el trabajo de Fingermann y Ramos (2009) quienes compilan todas las referencias sobre lalangue en los seminarios, escritos y conferencias de Lacan. ya que se superponen y se solapan entre ellos. No obstante, establecemos esta distinción para nuestros fines expositivos.

En primer lugar, respecto a los tropiezos del lenguaje, en "El Atolondradicho" (Lacan, 2018a), texto escrito en 1972, el psicoanalista francés relaciona a lalangue con el equívoco y, en este sentido, con el inconsciente. En este trabajo, el psicoanalista enfatiza que en su definición del inconsciente la preposición que emplea es "como": el inconsciente está estructurado como un lenguaje. Con ello, señala que el inconsciente no está estructurado "por" un lenguaje sino que, en cambio, habita la lalangue: "está sujeto al equívoco con la que cada una se distingue" (Lacan, 2018a, p. 514). De esta forma, el lenguaje no es un instrumento de comunicación unívoco; por el contrario, es "semblante de comunicación" (Lacan, 2018a, p. 515), en el que el lapsus, el sueño y el chiste tienen efectos. ${ }^{12}$ Entre estos equívocos, que se emplean en la interpretación, refiere a la homofonía, de la que el psicoanalista se sirve a lo largo de sus enseñanzas y de la que nace la misma noción de lalangue.

En el Seminario xx. Aún (2008), dictado entre 1972 y 1973, retoma la cuestión de la homofonía, en tanto que malentendido, al referirse al 'almor' (neologismo que reúne en su significante amor y alma) y a otra serie de términos homófonos:

Aquí debe ayudarme lalengua, la francesa, no ofreciéndome, como a veces ocurre, una homonimia entre d'eux y deux (...) sino simplemente permitiéndome decir que se alma. Yo almo, tú almas, él alma. Ven que aquí sólo nos sirve la escritura hasta para incluir nuncamás, nuncalmás. ${ }^{13}$ (Lacan, 2008, p. 102)

Lacan (1976a) distingue nuevamente el orden del lenguaje del orden de lalangue. Es en lalangue en la que anidan aliteraciones y rimas que dan cuenta de los equívocos y en la que se enraízan los sueños, los lapsus y los chistes. Al comienzo del Seminario XXIV. Lo no sabido que sabe de la una-equivocación

12 La visión del lenguaje como medio de comunicación vuelve a ser criticada en Psicoanálisis, radiofonía y televisión (Lacan, 1983).

13 Las cursivas son del original. 
se ampara en la morra (Lacan, 1976b) articula nuevamente estas tres formaciones, a las que denomina equívocos, con este término.

Como vemos, la relación entre lalangue y el equívoco se reitera en varios seminarios, conferencias y escritos: en efecto, lalangue es la integral de los equívocos que la distinguen. "En todo caso, lo que caracteriza lalengua son los equívocos posibles, como ilustré con el equívoco de deux [dos] con d'eux [de ellos]" (Lacan, 2018b, p. 115).

En segundo lugar, en cuanto al inconsciente, de vuelta al Seminario Xx. Aún, Lacan (2008) diferencia, una vez más, el lenguaje de lalangue. Aquí el lenguaje es definido como aquel que es elaborado por el discurso científico para dar cuenta de la comunicación, por lo que no tiene existencia propia: es aquello que "se procura saber respecto de la función de lalengua" (Lacan, 2008, p. 167). En cambio, lalangue es de lo que está hecha la experiencia del inconsciente, al que describe como su guarida. En otras palabras, mientras que el lenguaje es una elucubración de saber sobre lalangue, el inconsciente es un saber-hacer con ella. $\mathrm{Y}$ este saber-hacer excede lo que se puede decir, lo que se puede comunicar, lo que se soporta en el saber enunciado. Por eso, sus efectos son enigmáticos. El discurso analítico demuestra, en este sentido, que el lenguaje no es solamente comunicación.

El vínculo entre lalangue y el inconsciente vuelve a ser tratado, asimismo, en la conferencia "A la escuela freudiana” de 1974 y en la conferencia que dicta en Roma en ese mismo año y que lleva por título "La Tercera" (2007a). Por un lado, en la primera exposición recuerda que por el hilo de lalangue puede leerse la huella del saber del inconsciente. Por el otro, en la segunda señala que esta noción es condición del inconsciente y reitera que el saber del inconsciente se articula con ella.

En 1975 en la conferencia dictada en Ginebra Lacan (2007b) hace nuevamente alusión a lalangue $\mathrm{y}$ al inconsciente. En esta presentación se refiere al materialismo, para lo cual emplea, en francés, el neologismo moterialisme. A partir de esta noción condensa dos términos: materialismo (materalisme) y palabra (mot). Aquí el psicoanalista señala que el sustento del inconsciente se ubica en ese materialismo que es lalangue:

Es totalmente cierto que algo volverá a surgir luego en los sueños, en toda suerte de tropiezos, en toda suerte de maneras de decir, en función de la manera en que lalengua fue hablada y también escuchada por tal o cual en su particularidad. Es, si me permiten emplearlo por primera vez, en ese materialismo (materialismo de la palabra) dónde reside el asidero del inconsciente. ${ }^{14}$ (Lacan, 2007b, p. 126)

Por otra parte, además de relacionarse con el inconsciente, estos tropiezos del lenguaje se entienden también, como hemos afirmado, en función de la interpretación. En efecto, este vínculo ya aparece esbozado en el "Atolondradicho" (Lacan, 2018a), pero se retoma y se desarrolla dos años después en "La Tercera" (Lacan, 2007a). En esta conferencia, sostiene que lo real escribe en el síntoma y que el sentido lo alimenta. Por ello, afirma que la interpretación debe operar con lalangue, es decir, debe trabajar con los equívocos. De esta forma, no se trata de nutrir el síntoma de sentido, de consistir el síntoma a partir del sentido, sino, por el contrario, de deshacerlo a partir del equívoco, suspendiendo el sentido que porta:

En la medida en que, en la interpretación, la intervención analítica recae únicamente sobre el significante, algo del campo del síntoma puede retroceder. Aquí en lo simbólico, lo simbólico en tanto lo sostiene lalengua, se elabora el saber inscrito de lalengua que constituye propiamente el inconsciente, ganándole terreno al síntoma. (Lacan, 2007a p. 104)

En este fragmento se retoma la asociación entre el inconsciente y lalangue, y el lugar que ocupa la interpretación para que el síntoma retroceda.

Sobre el laleo y lalangue, es necesario, en primera instancia, indicar su relación con la lengua materna. En efecto, en el Seminario xx. Aún (Lacan, 2008) el psicoanalista francés hace referencia a que lalangue remite a lalangue materna:

14 La cursiva es del original. 
Lalengua sirve para otras cosas muy diferentes de la comunicación. Nos lo ha mostrado la experiencia del inconsciente, en cuanto está hecho de lalengua, esta lalengua que escribo en una sola palabra, como saber para designar lo que es el asunto de cada quien, la lengua llamada, y no en balde, materna. (Lacan, 2008, p. 166)

En esta cita se advierte que no hay una lalangue universal, sino que varía en función de cómo cada uno la recibe a través de esa primera "lengua materna".

En cuanto a la dimensión sonora y repetitiva presente en el laleo, ya en el Seminario XxI. Los incautos no yerran, dictado entre 1973 y 1974, en la clase del 8 de enero Lacan (s.f.) relaciona a lalangue con el ritornello. Recordemos que en términos musicales el ritornello es la repetición de un fragmento de una obra. En esta clase plantea además que en lalangue el sentido fluye copiosamente y, por ello, no queda anudado a ningún significante.

El laleo es mencionado explícitamente en la conferencia "A la escuela freudiana" del 30 de marzo de 1974, sobre la que ya hemos hecho referencia:

porque quiere decir lalala, la lalación, a saber, es un hecho que desde muy temprano el ser humano hace lalaciones, no hay más que ver a un bebé, escucharlo, poco a poco hay una persona, la madre, que es exactamente la misma cosa que lalangua, además es alguien encarnado quien le trasmite lalangua (sic). (Lacan, 1974, p. 24)

De esta cita se desprende que lalangue es singular, ya que es la madre quien la porta. Es ella quien la encarna y se la transmite al bebé. Podría pensarse que no solo le transmite palabras, sino inflexiones, tonos, afectos, sonidos, que forman parte de esta lalangue.

En la conferencia en Ginebra sobre el síntoma mencionada anteriormente, Lacan (2007b) vuelve sobre el laleo y aclara que el neologismo lalangue es lo más cercano al término francés lallation. Ubica, asimismo, a lalangue como un lenguaje que no tiene ninguna "existencia teórica" (Lacan, 2007b, p. 125), y que está en relación con cómo fue hablada y cómo fue escuchada por cada uno en su particularidad.

El 1 de diciembre de 1975, en una de las conferencias que tiene lugar en la Universidad de
Columbia sobre el síntoma, el psicoanalista recuerda que en el origen ya hay una relación con lalangue a la que hay que denominar "materna" porque el niño la recibe de su madre:

La experiencia consiste en esto, que desde el origen hay una relación con "lalengua", que merece ser llamada, justificadamente, materna, porque es por medio de la madre que el niño - si puedo decir - la recibe. Él no la aprende. Hay una inclinación. Es muy sorprendente ver cómo un niño manipula muy pronto algunas cosas tan notablemente gramaticales como el uso de las palabras "quizá" o "no todavía". Por supuesto, lo ha escuchado, pero que comprenda su sentido es algo que merece toda nuestra atención. (Lacan, 1975, p. 49)

A su vez, el vínculo entre lalangue y el laleo remite a su relación con el goce. Esta relación se destaca en "La Tercera" (2007a). Aquí el psicoanalista plantea que lalangue está hecha de goce y también que el goce se deposita en ella y la mortifica. Es por ello por lo que refiere a que lalangue, a pesar de que esté en uso, es una lengua muerta.

Finalmente, respecto del síntoma, es el eje que menos desarrolla en sus enseñanzas. El 16 de junio de 1975, al final de la conferencia "Joyce el Síntoma", Lacan asocia a lalangue con el goce del síntoma:

Soy lo bastante amo de lalengua, aquella llamada francesa, por haber alcanzado en ella lo fascinante de testimoniar respecto del goce del propio del síntoma. Goce opaco por excluir el sentido. (Lacan, 2018c, p. 596)

En este sentido, lalangue está articulado con el goce del síntoma, ella da testimonio de este goce.

Por último, en "El seminario de Caracas" (Lacan, 1982) señala que lalangue retoma este punto señalando que ella vehiculiza lo real. En efecto, no pude reducirse meramente a un juego de significantes.

\section{A modo de cierre}

En este artículo nos hemos interrogado respecto de las nociones de lengua, lenguaje y lalangue en las enseñanzas de Lacan. Hemos reflexionado, en primer lugar, sobre los motivos por los cuales Lacan 
utiliza el término lenguaje, en lugar de lengua, para hacer referencia al inconsciente a partir de revisar las posiciones teóricas acerca de este par en Saussure, Benveniste y Jakobson. En segundo lugar, hemos rastreado el neologismo lalangue en los escritos, seminarios y conferencias de este psicoanalista con el objetivo de analizar qué es lo que este aporta a sus enseñanzas.

Entre las conclusiones que hemos alcanzado destacamos que este trabajo nos ha permitido precisar que la noción de lenguaje es más próxima que el concepto de lengua a la conceptualización que Lacan realiza sobre el inconsciente. Mientras que la lengua remite a un sistema completo, homogéneo y delimitado, el lenguaje constituye un "hecho" complejo, heterogéneo y difícil de fijar. En este sentido, no puede reducirse a una única variable, ni clasificarse como categoría cerrada. Podría pensarse, en este panorama, que el lenguaje excede la lengua, por lo que es una noción que habilita al psicoanalista a configurar, en 1971, el término lalangue.

Por su parte, lalangue, como hemos visto, puede articularse con los equívocos, el inconsciente, la interpretación analítica, la lalación y el síntoma. Estas vertientes de la noción no son excluyentes; por el contrario, se interrelacionan y se complementan entre sí. Lalangue es un término que permite introducir la singularidad y la accidentalidad. En este punto, puede ubicarse en línea con el concepto saussureano de habla. Es aquello que irrumpe por fuera de la norma.

Su origen se halla en el laleo del bebé, repetición de un sonido que es puro goce. Este laleo no comunica, no porta un sentido, no arma sistema; es la madre quien puntúa allí, quien moldea y modela ese no-decir del bebé. Es esa lengua materna, lengua vernácula que no está estandarizada, reglada, que es propia de la madre, la que es transmitida al hijo. Y con ello, el afecto, el registro, la tonalidad, el ritmo propio.

Dahan (2011) plantea que mientras que el lenguaje unifica, lalangue es lo singular del sujeto. Por esta razón, asocia el lenguaje con la definición de lengua oficial y la lalangue con la de dialecto. Es el análisis, afirma la psicoanalista, lo que permite que el sujeto se autorice a hablar su propia lengua, por lo que tiene que pasar por un trabajo sobre lalangue.

Lalangue podría ser pensada, entonces, como un registro del lenguaje que interroga la unicidad a la que queda asociada la lengua. Es, en este punto, ese juego entre el deseo, el inconsciente y el goce que irrumpe en el decir no reglado, no normativizado, que emerge en el sujeto en esos espacios discursivos en los que los equívocos encuentran su materialidad y denotan el sin sentido que portan.

\section{Referencias}

Arrivé, M. (2000). Lacan gramático. Ágora, 3(2), 9-40.

Arrivé, M. (2001). Lingüística y psicoanálisis. Siglo XXI.

Benveniste, É. (2004a). Problemas de lingüística general. Tomo I y II. Siglo XXI.

Benveniste, É. (2004b). Ojeada al desenvolvimiento de la lingüística. En Problemas de lingüística general. Tomo I (pp.20-32). Siglo XXI.

Benveniste, É. (2004c). La forma y el sentido en el lenguaje. En Problemas de lingüística general. Tomo II (pp. 217-240). Siglo XXI.

Chomsky, N. (1997). Estructuras Sintácticas. Siglo XxI.

Dahan, P. (2011). Unité du langage, singularité de lalangue. Wunsch, 11, 30-34.

Fingermann, D. y Ramos, C. (2009). Lalíngua nos seminários, conferências e escritos de Jacques Lacan. Stylus: Revista de Psicanálise, 19. https://www.valas. fr/IMG/pdf/A_Lal-ngua_nos_semin-rios-_confer-ncias_e_escritos_de_Jacques_Lacan_-_OK.pdf

Flores, V. (1999). Linguística e psicanálise: princípios de uma semântica da enunciação. EDIPUCRS.

Godoy, C. (2016). Las resonancias de lalangue. En Actas del VIII Congreso Internacional de Investigación y Práctica Profesional en Psicología, XXIII Jornadas de Investigación, XII Encuentro de Investigadores en Psicología del Mercosur (pp.306-308). Facultad de Psicología Universidad de Buenos Aires, Buenos Aires.

Henry, P. (2012). Tirer lalangue. Essaim, 29, 97-109.

Henry, P. (2019). Acerca del equívoco. En J. AuthierRevuz, P. Henry y M. Arrivé. "Por más que Lacan lo diga”. Una introducción al Análisis del Discurso (pp. 67-97). Libretto. 
Jakobson, R. (1985). Lingüística y poética. En Ensayos de lingüística general (pp.347-395). Planeta Agostini.

Lacan, J. (1974, 30 de marzo). A la escuela freudiana. (M. Esnal, G. Leguizamón y M. C. Melegatti, trad.). http://ecole-lacanienne.net/wp-content/ uploads/2016/04/30-03-1974.pdf

Lacan, J. (1975, 24 de noviembre). Conferencias y charlas en universidades norteamericanas. (R. Rodríguez Ponte, trad.). https://www.lacanterafreudiana.com. ar/2.5.1.26\%20\%20\%20\%20CONFERENCIAS\%20 Y\%20CHARLAS\%20EN\%20UNIVERSIDADES\%20 NORTEAMERICANAS,\%201975.pdf

Lacan, J. (1976a, 24 de enero). De James Joyce como síntoma. Conferencia. https://www.lacanterafreudiana. com.ar/2.5.1.27\%20\%20\%20\%20DE\%20JAIMES\%20 JOYCE\%20COMO\%20SINTOMA,\%201976.pdf

Lacan, J. (1976b, 16 de noviembre- 1977, 17 de mayo). Seminario xxiv. Lo no sabido que sabe de la una-equivocación se ampara en la morra. http://www. bibliopsi.org/docs/lacan/29\%20Seminario\%2024.pdf

Lacan, J. (1982). El seminario de Caracas. http://www. psicoanalisis.org/lacan/caracas.htm

Lacan, J. (1983). Psicoanálisis, radiofonía y televisión. Editorial Anagrama.

Lacan, J. (1987). Seminario XI. Los cuatro conceptos fundamentales del psicoanálisis. Paidós.

Lacan, J. (2002). Función y campo de la palabra y del lenguaje en psicoanálisis. En Escritos 1 (pp. 231-309). Silgo XXI.

Lacan, J. (2007a). La Tercera. En Intervenciones y textos 2 (pp.73-108). Manantial.

Lacan, J. (2007b). Conferencia en Ginebra sobre el síntoma. En Intervenciones y textos 2. (pp.115-144). Manantial.
Lacan, J. (2008). Seminario xx. Aún. Paidós.

Lacan, J. (2012). Hablo a las paredes. Paidós.

Lacan, J. (2016). Seminario XIX. O peor. Paidós.

Lacan, J. (2018a). El Atolondradicho. En Otros escritos (pp. 473-521). Paidós.

Lacan, J. (2018b). Seminario XXIII. El sinthome. Paidós.

Lacan, J. (2018c). Joyce el Síntoma. En Otros escritos (pp. 591-597). Paidós.

Lacan, J. (s.f.). Seminario XXI. Los incautos no yerran. Inédito. www.psicoanalisis.org.

Miller, J.-A. (1987). Teoría de Lalengua. En Matemas I (pp.59-78). Buenos Aires: Ediciones Manantial.

Milner, J.-C. (1980). El amor por la lengua. Editorial Nueva Imagen.

Rouillon, J.P. (2011, 26 de noviembre). L'inconscient y lalangue. [Conferencia] Estrasburgo, Francia. http://www.lacan-universite.fr/wp-content/ uploads/2012/06/ROUILLON-J.-P.-18.pdf

Saussure, F. de (2007). Curso de linguística general. Tomo I y II. Losada.

Savio, K. (2015). Aportes de Lacan a una teoría del discurso. Folios, (42), 43-54 https://doi. org/10.17227/01234870.42folios43.54

Savio, K. (2017). El sujeto de la enunciación: diálogos entre la lingüística y el psicoanálisis. Linguagem em (dis)curso, 17(2), 271-284. http://dx.doi. org/10.1590/1982-4017-170207-2017

Savio, K. (2019). Sobre la noción de escritura en las enseñanzas de Lacan. Káñina, 43(1), 24-44. https://revistas.ucr.ac.cr/index.php/kanina/article/ view/36789 